\section{Right-sided endocarditis from Staphylococcus lugdunensis in a patient with tetralogy of Fallot}

\author{
Bradford Becken III, ${ }^{1}$ Jacob Kilgore, \\ Elizabeth Thompson, ${ }^{2}$ \\ M. Anthony Moody ${ }^{1}$ \\ ${ }^{1}$ Division of Pediatric Infectious \\ Diseases, ${ }^{2}$ Department of Pediatrics, \\ Duke University Children's Hospital, \\ Durham, NC, USA
}

\begin{abstract}
Infective endocarditis is often caused by bacterial pathogens and can affect native and prosthetic tissue. Common pathogens in pediatric patients include Staphylococcus aureus, viridans group streptococci, enterococcal species and coagulase-negative staphylococci, though culture-negative cases are not uncommon. Coagulase-negative staphylococci present a conundrum to clinicians due to the potential of culture contamination. While Staphylococcus lugdunensis is a coagulase-negative staphylococcus, it is an emerging cardiotropic pathogen that presents similarly to Staphylococcus aureus. Here we report a case of a child with repaired tetralogy of Fallot found to have right-sided infective endocarditis caused by Staphylococcus lugdunensis.
\end{abstract}

\section{Introduction}

Infective endocarditis (IE) is a significant concern in febrile patients with a history of prosthetic issue. Bacterial pathogens, predominantly Gram-positive organisms, are the most common cause of infective endocarditis, with Staphylococcus aureus, viridians group streptococci, and enterococcal species being the most common organisms. ${ }^{1}$ However, coagulase-negative staphylococci cause a significant proportion of infective endocarditis. Coagulase-negative staphylococci are grouped together based on their biochemical properties, but their ability to invade and destroy native tissue widely varies. Staphylococcus lugdunensis was first described in 1988, and while it is a coagulase-negative staphylococcus, it behaves more similarly to $S$. aureus than to the more commonly isolated coagulasenegative staphylococci $S$. epidermidis and $S$. hominis species, thus necessitating more aggressive management. ${ }^{2,3}$

\section{Case Report}

A 14-year-old male with a known history of tetralogy of Fallot (TOF) status post placement of a $10 \mathrm{~mm}$ right ventricle (RV) to pulmonary artery (PA) conduit at one year of age presented to the pediatric cardiologist for one month of fevers to $39^{\circ} \mathrm{C}$ and fatigue. Prior to evaluation, he had been treated with non-steroidal anti-inflammatory drugs (NSAIDs) as well as a one-week course of trimethoprim/sulfamethoxazole for a presumed urinary tract infection with only temporary relief of his symptoms. Over the resulting weeks, he developed a non-productive cough, non-bloody diarrhea, and worsening abdominal pain. He subsequently presented to the pediatric cardiologist for further evaluation despite being previously lost to subspecialty follow up for two years. No relevant social or exposure history was identified including denial of recent dental procedures or intravenous drug use. His exam was notable for a grade III/VI systolic ejection murmur loudest at the left upper sternal border with radiation to both axillae, documented to be louder than previous exams. There was no evidence of splinter hemorrhages, retinal hemorrhages, or distal cutaneous findings consistent with sequelae of infective endocarditis. The patient underwent a transthoracic echocardiogram that showed multiple vegetations in the right ventricular outflow tract, pulmonary valve, and PA conduit. The largest vegetation measured $20 \times 12 \mathrm{~mm}$ with smaller vegetations measuring $9 \times 9 \mathrm{~mm}$ and $7 \times 3 \mathrm{~mm}$, respectively. Subsequent chest CT imaging re-demonstrated cardiac vegetations in addition to revealing bilateral pulmonary nodules concerning for septic emboli (Figure 1).

He was admitted to the hospital and pediatric infectious diseases was consulted. The patient was empirically started on intravenous vancomycin and gentamicin therapies. Initial labs were significant for a Creactive protein (CRP) of $13.78 \mathrm{mg} / \mathrm{dL}$ (reference range $<0.60 \mathrm{mg} / \mathrm{dL}$ ), erythrocyte sedimentation rate (ESR) of $34 \mathrm{~mm} / \mathrm{hr}$ (reference range $0-13 \mathrm{~mm} / \mathrm{hr}$ ), a creatinine of $0.9 \mathrm{mg} / \mathrm{dL}$ (reference range $0.3-0.8 \mathrm{mg} / \mathrm{dL}$ ), a sodium of $127 \mathrm{mmol} / \mathrm{L}$ (reference range $135-145 \mathrm{mmol} / \mathrm{L}$ ), and an INR of 1.4. The white blood cell count was normal; however, the differential revealed a neutrophilic predominance with mild bandemia. Vancomycin troughs were kept between 11.5 and $14.5 \mu \mathrm{g} / \mathrm{mL}$. Blood cultures ultimately grew pan-sensitive Staphylococcus lugdunensis. On hospital day 6, the patient was transitioned to intravenous cefazolin $150 \mathrm{mg} / \mathrm{kg} /$ day and rifampin $600 \mathrm{mg} /$ day due to lack of central access preventing continuous nafcillin use.
Correspondence: Bradford Becken, Division of Pediatric Infectious Diseases, Duke University Medical Center, T915 Children's Health Center, Box 3499 Durham, NC 27710, USA.

Tel: +1.919-684-6335 - Fax: +1.919-668-4859.

E-mail: bradford.becken@duke.edu

Key words: Staphylococcus lugdunensis, coagulase negative staphylococcus, endocarditis, pediatric and tetralogy of Fallot.

Contributions: BB provided direct patient care for the subject, conceptualized case report, performed literature review and drafted and edited the manuscript; JK provided direct patient care for the subject, conceptualized case report with $\mathrm{BB}$ and ET, performed literature review and drafted and edited the manuscript; ET provided direct patient care for the subject (inpatient pediatric cardiology service), assisted with conceptualization of case report and literature review; she also help draft manuscript and provided editing support. MAM provided direct patient care for the subject (pediatric infectious diseases consult), assisted with literature review and drafting of manuscript as well as editing support, he also helped review applicable case report guidelines.

Conflict of interest: the authors declare no potential conflict of interest.

Funding: none.

Received for publication: 16 September 2018 Revision received: 4 November 2018.

Accepted for publication: 12 February 2019.

This work is licensed under a Creative Commons Attribution-NonCommercial 4.0 International License (CC BY-NC 4.0).

(C) Copyright B. Becken III et al., 2019

Licensee PAGEPress, Italy

Infectious Disease Reports 2019; 11:7872

doi:10.4081/idr.2019.7872

Despite the aforementioned antibiotic treatment, blood cultures remained persistently positive for 10 days. On hospital day 11 , he underwent cardiothoracic operative repair in addition to central line placement. Per the intraoperative report, the anterior portion of the conduit wall was compromised by infection, leaving the conduit thinned and with a pseudo-aneurysm formation. Additionally, the conduit valve had been destroyed. The compromised portions of the conduit were removed and vegetations were debrided. A $25 \mathrm{~mm}$ pulmonary homograph was installed in place of the old conduit with a portion of the re-anastomosis being augmented with de-cellularized 
bovine pericardium (CardioCel). The patient tolerated the procedure well and was extubated in the operating room. Following surgical intervention and central line placement the patient was transitioned to continuous infusion nafcillin monotherapy with clearance of repeat blood cultures. Prior to discharge the patient operative pace right internal jugular line was removed and a peripherally inserted central catheter (PICC) was placed. He was discharged home 6 days after surgical intervention to complete a planned 6-week course of a nafcillin 8 grams/day via continuous infusion, which based on the patient's weight, was $175 \mathrm{mg} / \mathrm{kg} /$ day. Though the patient clinically improved during his planned course, owing to persistently elevated inflammatory markers after 6-weeks of therapy, the nafcillin course was extended for an additional two weeks for a total of eight weeks of therapy. At his 8-week outpatient follow up with pediatric infectious diseases, inflammatory markers had normalized and his clinical examination was reassuring. A follow up echocardiogram demonstrated normal cardiac function and no residual vegetations. The patient's PICC line was removed, and he continued with close interval follow up with pediatric cardiology.

\section{Discussion}

Infective endocarditis (IE) occurs when there is an infection of the cardiac endothelium or prosthetic material associated with the heart. Bacterial pathogens are implicated in the majority of cases, with Staphylococcus aureus, viridians group streptococci, and enterococcal species being the most common organisms isolated - though pathogens do vary by patient age and underlying cardiac disease. ${ }^{1}$ However, coagulase-negative staphylococci cause a

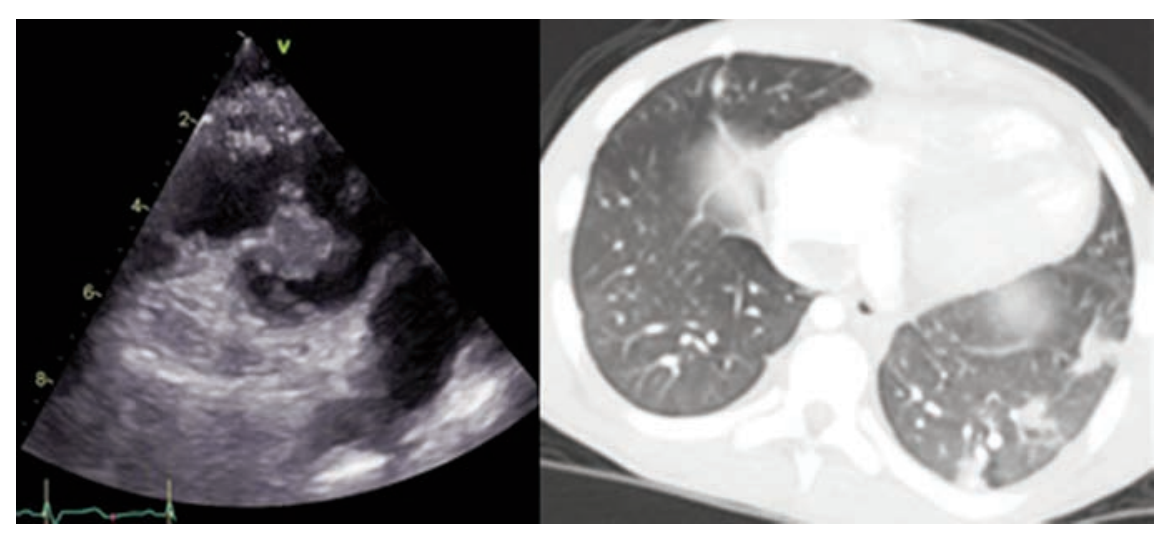

Figure 1. Transthoracic echocardiogram demonstrating the largest mobile mass on pulmonary valve $(20 \times 12 \mathrm{~mm})$ and companion chest computed tomography showing subsequent pulmonary emboli. significant proportion of infective endocarditis of both native and prosthetic valves. Though coagulase-negative staphylococci are grouped together based on their biochemical properties, their ability to invade and destroy native tissue widely varies with S. lugdunensis known to be more aggressive than the more commonly isolated $S$. epidermidis and $S$. hominis species. Characterized in 1988, S. lugdunensis is believed to have been previously misidentified as $S$. aureus because it is able to produce clumping factor and a positive DNase test. Additionally, its colony morphology closely resembles that of $S$. aureus. ${ }^{2,3}$

Reports of S. lugdunensis infections are rare, especially regarding infective endocarditis in pediatric patients. ${ }^{4-6}$ One hypothesis regarding the dearth of pediatric cases is that colonization of the skin from $S$. lugdunensis occurs later in life. ${ }^{7}$ Another reason for the paucity of cases is that given the rarity of the organism, even when solely compared to other species of coagulasenegative staphylococci, it may be erroneously regarded as a contaminant. ${ }^{3,8}$ Despite the aggressive nature of $S$. lugdunensis, it is typically more sensitive to $\beta$ lactams than other strains of coagulase-negative staphylococci. However, as was the case with this patient, surgical intervention is often required. In a retrospective review of patients with IE from The Cleveland Clinic, patients with $S$. lugdunensis, when compared to those with $S$. aureus, were more likely to have bulky vegetations, and the affected patients were more likely to have had native valves but with pre-existing cardiac disease. Additionally, $13 \%$ of all isolates of $S$. lugdunensis were from patients who met the modified Duke's criteria for IE. ${ }^{8}$

As with any patient with prosthetic cardiac material, prompt evaluation of unex-

plained fevers is necessary. Presenting

To help prevent similar occurrence, our case emphasizes the importance of routine specialized care in patients with complex cardiac disease

\section{References}

1. Baltimore R, Gewitz M, Baddour L, et al. Infective Endocarditis in Childhood: 2015 Update. Circulation. 2015;132;1487-15.

2. Hellbacher C, Tornqvist E, Soderquist C. Staphylococcus lugdunensis: clinical spectrum, antibiotic susceptibility, and phenotypic and genotypic patterns of 39 isolates. Clin Microbiol Infect 2006;12:43.49. 
3. Argemi X, Hansmann Y, Riegel P. Prévost G. Is Staphylococcus lugdunensis Significant in Clinical Samples? J Clin Microbiol 2017;55:3167-74.

4. Anguera I, Del Río A, Miró JK, et al. The Hospital Clinic Endocarditis Study Group. Staphylococcus lugdunensis infective endocarditis: description of 10 cases and analysis of native valve, prosthetic valve, and pacemaker lead endocarditis clinical profiles. Heart 2005;91:040659.

5. Vandenesch F, Etienne J, Reverdy ME, Eykyn, SJ. Endocarditis Due to
Staphylococcus lugdunensis: Report of 11 Cases and Review. Clin Infect Dis 1993;17:871-6.

6. Patel R, Piper KE, Rouse MS, et al. Frequency of Isolation of Staphylococcus lugdunensis among Staphylococcal Isolates Causing Endocarditis: a 20-Year Experience. J Clin Microbiol 2000:4262-3.

7. Herchline TE, Ayers LW. Occurrence of Staphylococcus lugdunensis in Consecutive Clinical Cultures and Relationship to Isolation of Infection. J Clin Microbiol 1991:419-21.
8. Sabe M, Shrestha N, Gordon S, Menon V. Staphylococcus lugdunensis: a rare but destructive cause of coagulase-negative staphylococcus infective endocarditis. Eur Heart J 2014;3:275-880.

9. Holland T, Baddour L, Bayer A, et al. Infective endocarditis. Nat Rev Dis Primers 2016;2:16059.

10. Ferrieri P, Gewitz M, Gerber M, et al. Unique Features of Infective Endocarditis in Childhood. Circulation 2002;105:2115-26. 